\title{
NOTIFICAÇÃO COMPULSÓRIA DE AGRAVOS EM SAÚDE DO TRABALHADOR: AVALIANDO A SUBNOTIFICAÇÃO
}

\author{
COMPULSORY NOTIFICATION OF AGGRAVATIONS IN \\ WORKERS HEALTH: EVALUATING SUBNOTIFICATION
}

Anna Neri Batista da Silva (Orcid: 0000-0001-7819-6021) ${ }^{1}$ Lourdes Auxiliadora de Oliveira (Orcid: 0000-0002-2234-0028) ${ }^{1}$ Jessica Carvalho Lima (Orcid: 0000-0003-0972-1886)

Patricia Ribeiro Marcacine (Orcid: 0000-0003-1784-2231) ${ }^{2}$

Ana Carolina Silva Ribeiro (Orcid: 0000-0002-4953-3268)

Gabriela Rodrigues (Orcid: 0000-0002-4953-3268) ${ }^{3}$

Isabel Aparecida Porcatti de Walsh1 (Orcid: 0000-0002-2317-1326)

\section{RESUMO}

Apesar dos avanços ocorridos na Saúde do Trabalhador, como a criação da Política Nacional de Saúde do Trabalhador e da Trabalhadora e a portaria de notificação compulsória dos agravos e doenças relacionadas ao trabalho, o presente ensaio teórico busca contextualizar os porquês da subnotificação presente na realidade profissional. Ressalta a importância de mudanças efetivas nas grades curriculares na formação de futuros profissionais, a falta de conhecimento de várias profissões, sobre a temática, a preferência pela utilização do modelo assistencialista e a falta de interesse dos especialistas da saúde em preencherem as notificações. Nesse sentido, cabe a reflexão sobre a importância de haver mais efetivação da política de Saúde do Trabalhador e comprometimento dos profissionais para mudanças no contexto da realidade em saúde do trabalhador.

Palavras-chave: Notificação compulsória; Saúde do trabalhador; Doenças profissionais.

\section{Autor correspondente:}

Isabel Aparecida Porcatti de Walsh

E-mail: isabelpwalsh@gmail.com

\footnotetext{
${ }^{1}$ Programa de Pós-graduação em Fisioterapia. Universidade Federal do Triângulo Mineiro e

Universidade Federal de Uberlândia (UFU).

${ }^{2}$ Programa de Pós-graduação em Atenção à Saúde.

Universidade Federal do Triângulo Mineiro

${ }^{3}$ Curso de Fisioterapia. Universidade Federal

do Triângulo Mineiro

${ }^{4}$ Departamento de Fisioterapia Aplicada.

Universidade Federal do Triângulo Mineiro
}

\begin{abstract}
Despite advances in Occupational Health, such as the creation of the National Occupational Health Policy and the mandatory notification of work-related injuries and illnesses, this theoretical essay seeks to contextualize the reasons for underreporting present in the professional reality. It highlights the importance of effective changes in the curriculum in the training of future professionals, the lack of knowledge of various professions on the subject, the preference for using the assistance model and the lack of interest of health specialists in filling out notifications. In this sense, it is worth reflecting on the importance of making the Occupational Health policy more effective and the professionals' commitment to changes in the context of the reality in occupational health.
\end{abstract}

Keywords: Disease notification; Occupational health; Occupational diseases. 


\section{POLÍTICAS PÚBLICAS EM SAÚDE DO TRABALHADOR}

Desde o século XVIII, com a Revolução Industrial na Inglaterra, houve modificação dos processos de trabalho, com condições e ambientes impróprios ocasionando acidentes e adoecimento dos trabalhadores, desencadeando prejuízos sociais e econômicos, sendo necessário pensar uma forma diferenciada de atenção à saúde dos trabalhadores ${ }^{1}$. A medicina do trabalho, enquanto especialidade médica, surgiu na Inglaterra, na primeira metade do século XIX, com a Revolução Industrial $^{2}$, em que o médico era contratado para estar presente nas indústrias e se responsabilizar pela saúde dos trabalhadores ${ }^{1}$.

Em um contexto econômico e político como o da guerra e o do pós-guerra, o custo provocado pela perda de vidas por acidentes e doenças do trabalho passou a ser sentido pelos empregadores e companhias de seguro, revelando a impotência da medicina do trabalho para intervir sobre os problemas de saúde causados pelos processos de produção. A resposta foi traduzida na ampliação da atuação médica direcionada ao trabalhador, pela intervenção sobre o ambiente, surgindo a Saúde Ocupacional, dando-se dentro das grandes empresas, com o traço da multi e interdisciplinaridade, com a organização de equipes progressivamente multiprofissionais e a ênfase na higiene industrial ${ }^{3 .}$

A insuficiência do modelo da saúde ocupacional se desenvolveu em cenários políticos e sociais amplos e complexos, em um movimento social renovado, que surgiu nos países industrializados do mundo ocidental na segunda metade da década de 1960, com o questionamento do sentido da vida, o valor da liberdade, o significado do trabalho na vida, o uso do corpo, e a denúncia de valores sem significado para a nova geração, que abalaram a confiança no Estado, levando alguns países à exigência da participação dos trabalhadores nas questões de saúde e segurança ${ }^{4,5}$.
Como resposta ao movimento social e dos trabalhadores, novas políticas sociais se transformaram em leis, com significativas mudanças na legislação do trabalho e nos aspectos de saúde e segurança do trabalhador, tendo como exemplo a Itália; e, de natureza semelhante, com algumas peculiaridades próprias de contextos político-sociais distintos, foram também sendo alcançados pelos trabalhadores norte-americanos, ingleses, suecos, franceses, noruegueses, canadenses, entre outros $^{6-11}$.

Essa nova legislação teve como pilares comuns o reconhecimento do exercício de direitos fundamentais dos trabalhadores, entre eles: o da informação sobre a natureza dos riscos, as medidas de controle que estão sendo adotadas pelo empregador, os resultados de exames médicos e de avaliações ambientais, e outros; o da recusa ao trabalho em condições de risco grave para a saúde ou a vida; o da consulta prévia aos trabalhadores, antes de mudanças de tecnologia, métodos, processos e formas de organização do trabalho e o estabelecimento de mecanismos de participação, desde a escolha de tecnologias até, em alguns países, a escolha dos profissionais que irão atuar nos serviços de saúde no trabalho $0^{6,9-13}$.

\section{O movimento pela Reforma}

Sanitária Europeia influenciou as manifestações no Brasil, na década de 1970, com reivindicações principalmente por parte dos trabalhadores, organizados e apoiados pelo sindicalismo, para a Reforma Sanitária Brasileira, incluindo questões de Saúde do Trabalhador (ST), buscando o direito pelo trabalho digno e saudável, a opinião dos trabalhadores na organização da produção e a assistência à saúde ${ }^{1}$.

No Brasil, as iniciativas pela criação de locais que acolhessem os trabalhadores se deram por meio da instituição de Programas de ST, com o pioneirismo do estado de São Paulo, onde também surgiram os primeiros Centros de Referência em ST, com caráter assistencial, antes do Sistema Único de Saúde (SUS), 
tomando forma o movimento da ST no País. Em 1986, aconteceu a VIII Conferência Nacional de Saúde e a I Conferência Nacional de ST em que foram elencadas as condições dignas de trabalho e a participação dos trabalhadores na definição dos processos e ambientes de trabalho como fatores imprescindíveis para o acesso pleno à saúde, sendo essa direito de todos e dever do Estado, modificando a perspectiva da classe trabalhadora. A ST se consolida no plano legal e institucional a partir da Constituição Federal de 1988 e com a posterior Lei 8.080, de $1990^{14}$.

Na década de 1990, aconteceram alguns marcos importantes, como a II Conferência Nacional de ST que reiterou a necessidade de municipalização das ações de ST, a instituição da Comissão Intersetorial de Saúde do Trabalhador e da Trabalhadora (CISTT), a criação da Norma Operacional de ST (Nost/SUS), legislação orientadora da gestão, elaborada a Lista de Doenças Relacionadas ao Trabalho, publicação de protocolos, normas e diretrizes norteadoras para execução das ações ${ }^{14}$.

A década de 2000 trouxe à ST a proposta de criação da Rede Nacional de ST que se efetivou no ano de 2003, a qual se integrou à rede de serviços do SUS por meio de Centros de Referência em Saúde do Trabalhador (Cerest). Em 2005, na III Conferência Nacional de ST, houve a reflexão sobre a criação da Política Nacional de ST, que foi elaborada, colocada em consulta pública $^{14}$.

Apesar dos avanços ocorridos no campo da ST, apenas em 2011, criou-se a Política Nacional de Segurança e Saúde no Trabalho (PNSST) e, tão somente em 2012, foi publicada a Política Nacional de Saúde do Trabalhador e da Trabalhadora (PNSTT) $)^{15,16}$.

A PNSST tem por objetivos a promoção da saúde e a melhoria da qualidade de vida do trabalhador e a prevenção de acidentes e de danos à saúde advindos do trabalho ou que ocorram no curso dele, por meio da eliminação ou redução dos riscos nos ambientes de trabalho. Ao mesmo tempo, estabelece uma rede integrada de informações em ST ${ }^{17}$. Esta enfatiza a vigilância à saúde como estratégia para a promoção e a proteção da saúde dos trabalhadores e para a redução da morbimortalidade decorrente dos modelos de desenvolvimento e dos processos produtivos ${ }^{16}$.

Suas estratégias envolvem a articulação das ações gerais das vigilâncias epidemiológicas, ambientais e sanitárias à categoria trabalho, considerando-as como um dos determinantes do processo saúde-doença dos indivíduos e da coletividade. Destacam-se nela a apresentação das responsabilidades do Ministério do Trabalho e Emprego (MTE), Ministério da Saúde (MS) e Ministério da Previdência Social (MPS), no desenvolvimento das ações de ST. Busca-se, também, fortalecer a Vigilância em Saúde do Trabalhador (Visat) por meio da integração dos demais componentes da vigilância em saúde. Portanto a PNSTT tem como finalidade definir os princípios, as diretrizes e as estratégias a serem observados nas três esferas de gestão do SUS - federal, estadual e municipal -, para o desenvolvimento das ações de atenção integral à ST, com ênfase na vigilância, visando à promoção e à proteção da saúde dos trabalhadores e à redução da morbimortalidade decorrente dos modelos de desenvolvimento e dos processos produtivos ${ }^{16}$.

Definem-se como sujeitos desta política: todos os trabalhadores, independentemente de sua localização, forma de inserção no mercado de trabalho ou seu vínculo empregatício, que representam sujeitos de mudanças nas práticas profissionais. A política segue os princípios e diretrizes do SUS, atentando-se principalmente para a garantia da atenção integral à ST, em todos os pontos das Redes de Atenção à Saúde (RAS) do SUS, o que inclui a construção de protocolos, 
linhas de cuidados e matriciamento na assistência e organização da rede ${ }^{16}$.

A Rede Nacional de Atenção Integral à Saúde do Trabalhador (Renast) é considerada como a principal estratégia para a implementação da PNSTT no SUS, estando estruturada a partir dos Cerest de abrangência estadual, regional ou municipal, apresentando como atribuições: I - desempenhar as funções de suporte técnico, de educação permanente, de coordenação de projetos de promoção, vigilância e assistência à saúde dos trabalhadores, no âmbito da sua área de abrangência; II - dar apoio matricial para o desenvolvimento das ações de ST na atenção primária em saúde, nos serviços especializados e de urgência e emergência, bem como na promoção e vigilância nos diversos pontos de atenção da RAS; e III - atuar como centro articulador e organizador das ações intra e intersetoriais de ST, assumindo a retaguarda técnica especializada para o conjunto de ações e serviços da rede SUS e se tornando polo irradiador de ações e experiências de vigilância em saúde, de caráter sanitário e de base epidemiológica ${ }^{16}$.

Enquanto instância da Renast, o Cerest desempenha função de suporte técnico, de educação continuada, de coordenação de projetos de Assistência, Promoção e Vigilância à ST no âmbito de sua abrangência, assumindo papel de Apoio Matricial para o Desenvolvimento das ações de ST na Assistência à Saúde e Vigilância em Saúde no estado, sendo um Centro Articulador e Organizador das ações Intra e Intersetoriais de ST, assumindo a Retaguarda Técnica e se tornando polo irradiador de ações e experiências de vigilância em saúde, de caráter sanitário e base epidemiológica, dando suporte aos municípios que compõem suas abrangências para a operacionalização da PNSTT no SUS e fortalecimento da RENAST ${ }^{17}$.

A composição mínima de sua equipe técnica é de 6 profissionais de nível superior, sendo: 2 médicos/20 horas semanais; 1 enfermeiro/40 horas semanais; 3 profissionais de nível superior de outras categorias (engenheiro, tecnólogo, médico veterinário, farmacêutico, fisioterapeuta, odontólogo, terapeuta ocupacional, psicólogo, fonoaudiólogo, assistente social, entre outros) - e mais 4 profissionais de nível médio, sendo: 2 auxiliares de enfermagem; 2 profissionais de nível médio (técnico de segurança do trabalho e auxiliar de enfermagem do trabalho, entre outros) $)^{18}$.

\section{ADOECIMENTO NO TRABALHO}

De acordo com a Norma Regulamentadora no 9 (NR-9) que trata do Programa de Prevenção aos Riscos Ambientais (PPRA), do MTE, os riscos ambientais são divididos em agentes físicos, biológicos, químicos, ergonômicos e de acidente. Esses riscos são inerentes ao ambiente laboral; e que, em função de sua natureza, intensidade e tempo de exposição, são capazes de prejudicar a $\mathrm{ST}^{19}$.

Mundialmente, as doenças e acidentes relacionados ao trabalho se verificam como desafio para a saúde pública, e um grande problema a ser solucionado é a captação de informações oficiais sobre essas ocorrências. Cada país opta por formas de apurar esses dados, e geralmente o levantamento de acidentes e doenças é aquém dos números reais e remetem aos trabalhadores formais, não refletindo a totalidade da população trabalhadora ${ }^{20}$.

No Brasil, há um alto número de acidentes e doenças relacionados ao trabalho. De acordo com dados do Observatório Digital de Saúde e Segurança do Trabalho, de 2012 a 2018, o Brasil registrou 16.455 mortes e 4,5 milhões de acidentes. No mesmo período, foram perdidos 351,7 milhões de dias de trabalho com afastamentos previdenciários e acidentários ${ }^{21}$. 


\section{NOTIFICAÇÃO COMPULSÓRIA DE AGRA- VOS E DOENÇAS RELACIONADAS AO TRABALHO}

Ao se estabelecer o nexo entre a doença/ acidente do trabalho, faz-se necessário o registro oficial, com a emissão da Comunicado de Acidente de Trabalho (CAT) ao Instituto Nacional do Seguro Social (INSS), visando a garantia do acesso ao benefício auxílio-doença acidentário, garantindo 12 meses de estabilidade na empresa ao trabalhador que se afastou por mais de 15 dias, quando retornar ao trabalho, entre outros. A CAT é uma importante fonte de informações epidemiológicas, embora se refiram apenas aos trabalhadores cobertos pelo Seguro Acidente de Trabalho, sendo, portanto, excluídos dessa fonte de dados os trabalhadores autônomos, domésticos, funcionários públicos estatutários, subempregados, muitos trabalhadores rurais, entre outros ${ }^{22,23}$.

No entanto, considerando os dados do levantamento feito a partir dos microdados da Pesquisa Nacional por Amostra de Domicílios Contínua (PNAD Contínua) - de que 62,4\% das pessoas que trabalham na informalidade estão no interior, contemplando 20,8 milhões de trabalhadores sem carteira assinada (empregados do setor privado e trabalhadores domésticos), sem CNPJ e sem contribuição para a previdência oficial (empregadores e por conta própria) ou sem remuneração (auxiliam em trabalhos para a família), sendo que $36,3 \%$ da população ocupada está em uma dessas condições de informalidade ${ }^{24}$ -, fazem-se necessários outros instrumentos para que os números estatísticos correspondam à realidade de toda a população trabalhadora.

Nesse sentido, a notificação compulsória é a comunicação da ocorrência de determinadas doenças ou agravos à saúde por parte dos profissionais de saúde à autoridade sanitária.
É obrigatória a todos os profissionais de saúde no exercício da profissão, bem como os responsáveis por organizações e estabelecimentos públicos e particulares de saúde ${ }^{25}$, devendo ser feita para qualquer trabalhador, independentemente de seu vínculo de trabalho.

Desde 2004, o Ministério da Saúde tornou obrigatória a notificação de 11 agravos e doenças relacionadas ao trabalho, atualmente em vigor por meio da Portaria GM/MS 20426, sendo estes: Acidentes de Trabalho Fatais, Graves e com Crianças e Adolescentes; Acidente de Trabalho com Exposição à Material Biológico; Lesões por Esforços Repetitivos/Distúrbios Osteomusculares Relacionados ao Trabalho, LER/ DORT; Perda Auditiva Induzida por Ruído Ocupacional; Câncer Relacionado ao Trabalho; Pneumoconiose; Dermatose Ocupacional; Transtorno Mental Relacionado ao Trabalho e Intoxicação Exógena ${ }^{26}$.

No entanto, há grande subnotificação do registro desses agravos no Sistema de Informações de Agravos de Notificação (Sinan), uma vez que seu número é muito baixo quando comparado ao de países desenvolvidos ${ }^{28}$. Existe o desconhecimento por parte dos profissionais de saúde acerca do trabalho como importante determinante do processo saúde/doença e da importância dos agravos à saúde relacionados ao trabalho, o que desencadeia a falta de priorização na identificação e notificação dessas ocorrências. Entre os aspectos mais relevantes com relação às subnotificações, mencionam-se as dúvidas apresentadas pelos profissionais na identificação dos agravos, o desconhecimento e a indefinição do papel de cada um na identificação dos acometimentos relacionados ao trabalho e consequente notificação, o trabalho em equipe insuficiente e a desvalorização da notificação por alguns profissionais, vinculada ao desconhecimento da finalidade desses dados. Ainda, aspectos observados na formação de profissionais de saúde para atuarem na rede privada podem influenciar na relevância às questões de saúde pública, como essas $^{27}$. 
Dessa maneira, o papel das Instituições acadêmicas, sobretudo das universidades públicas, é relevante na formação integral e de qualidade sobre ST. Alguns desafios devem ser vencidos, como ampliação dessa temática nos cursos de graduação que formam os futuros profissionais de saúde que serão os executores dessa política na rede SUS ou nos estabelecimentos de saúde privados. Para isso, faz-se necessária a seleção de docentes aptos a ministrar tal disciplina, cumprindo ementas pertinentes a essa abordagem e com capacidade persuasiva, influenciando os discentes, dos primeiros anos ao período de estágio, a realizarem atividades voltadas à ST, desde a prevenção de adoecimentos e acidentes no trabalho até a identificação destes e preenchimento das notificações compulsórias.

Ainda, a ficha de notificação compulsória deve ser preenchida com qualidade, tornando possível a utilização dos dados obtidos no Sinan, disponibilizando informações relevantes na construção de políticas de saúde, como a identificação das condições de ST, e planejamento de intervenções, como vigilância da situação de saúde dos trabalhadores e dos ambientes e processos de trabalho. Para garantia de dados fidedignos necessários, é essencial a educação continuada em ST e notificação compulsória aos profissionais; bem como a completitude dos campos das fichas de notificação ${ }^{20}$.

A escassez e a inconsistência das informações sobre a real situação de saúde dos trabalhadores dificultam a definição de prioridades para as políticas públicas, o planejamento e a implementação das ações de ST, além de privar a sociedade de subsídios importantes para a melhoria das condições de vida e trabalho ${ }^{28}$. Já o estudo desses agravos tem-se mostrado de importância fundamental no cenário das atividades de vigilância em saúde, pois permite a realização do diagnóstico da ocorrência do evento na população, fornecendo subsídios para explicações causais, além de indicar os riscos aos quais as pessoas estão sujeitas, contribuindo para a identificação da realidade epidemiológica de determinada área geográfica. Portanto, esse é um instrumento relevante para auxiliar o planejamento da saúde, definir prioridades de intervenção, avaliar o impacto das intervenções e subsidiar ações no campo da ST, especialmente no planejamento e organização dos serviços e na proposição de estratégias de educação e prevenção $0^{29}$.

Assim, a ausência ou o baixo número de registros dos agravos à saúde relacionados ao trabalho, nos sistemas de informação do SUS, dificultam a visibilidade social dos problemas e sua inserção nas agendas técnica e política dos gestores e conselhos de saúde ${ }^{30}$, acarretando entraves no SUS, como a manutenção e/ou criação de políticas públicas que promovam intervenção nas ocorrências de tais acometimentos, seja para evitar uma epidemia, ou até mesmo para criar o perfil epidemiológico da população, ajudando na regulação de recursos e atenção especial a certas populações vulneráveis a doenças e agravos específicos ${ }^{27}$.

Dessa maneira, a melhoria desses dados é um desafio assumido nacionalmente para incluir as questões da ST em todas as instâncias do SUS ${ }^{30}$.

\section{CONSIDERAÇÕES FINAIS}

Finalmente, verifica-se que o campo da ST no Brasil apresentou avanços importantes nas últimas décadas. No entanto, alguns desafios devem ser vencidos para que se tenha maior fidedignidade quanto à epidemiologia dos adoecimentos no trabalho. Um entrave a ser vencido é o despertar dos profissionais que já estão atuando nos serviços de saúde para que se interessem pela causa da ST, mesmo porque são também trabalhadores; e entendendo o processo trabalho/saúde/doença, serão os protagonistas em promover a própria saúde e a da coletividade que assistem. Esses são 
imprescindíveis para dar visibilidade às ocorrências de acidentes e adoecimentos em virtude do trabalho, exercendo o dever legal de agentes que devem notificá-los, possibilitando a manutenção e a ampliação das Políticas Públicas que garantam a atuação dos serviços de saúde, no sentido de prevenir novas ocorrências e intervir nos processos e ambientes de trabalho, tornando o trabalho mais seguro e com condições dignas aos trabalhadores.

\section{REFERÊNCIAS}

1. Dias EC, Hoefel M da G. O desafio de implementar as ações de saúde do trabalhador no SUS: a estratégia da RENAST. Ciênc Saúde Coletiva 2005; 10(4):817-27.

2. Schilling RSF. Developments in occupational health. 2nd ed. Butherworths, London 1981; 3-26 p.

3. Mendes R, Dias EC. Da medicina do trabalho à saúde do trabalhador. Rev Saúde Pública 1991; 25(5):341-9.

4. Berlinguer G. A Saúde nas fábricas. Ceber / Hucitec; 1978.

5. Rigotto RM. A saúde do trabalhador como campo de luta. Belo Horizonte; 1990.

6. Assennato G, Navarro V. Workers' Participation and Control in Italy: The Case of Occupational Medicine: Int J Health Serv [Internet] 1995 [acessado $2020 \mathrm{fev} 14]$; Disponível em: https://journals.sagepub.com/ doi/10.2190/QE4G-6984-J1RJ-EJVG

7. Bagnara S, Biocca M, Mazzonis DG. Trends in occupational health and safety policy in Italy. Int J Health Serv Plan Adm Eval 1981; 11(3):431-50.

8. Mendes R. A prática da integração da saúde ocupacional no setor saúde: análise de alguns modelos estrangeiros. Rev bras Saúde ocup 1988; 17(65):7-15.

9. Parmeggiani LA. A nova legislação de segurança e saúde dos trabalhadores. Rev bras Saúde ocup 1985; 15(50):19-27.

10. Reich MR, Goldman RH. Italian occupational health: concepts, conflicts, implications. Am J Public Health 1984; 74(9):1031-41.

11. Roustang G. Os trabalhadores e a higiene e segurança na França. Rev bras Saúde ocup 1985; 13(5):40-6.

12. Baetjer AM. The early days of Industrial Hygiene - their contribution to the current problems. Am Ind Hyg Assoc J 1980; (41):773-7.

13. Oddone I. Ambiente de trabalho: a lutados trabalhadores pela saúde. São Paulo: Hucitec; $1986.133 \mathrm{p}$.

14. Gomez CM, Vasconcellos LCF, Machado JMH. Saúde do trabalhador: aspectos históricos, avanços e desafios no Sistema Único de Saúde. Ciênc Saúde Coletiva 2018; 23(6):1963-70.

15. Brasil. Casa Civil. Decreto no 7.602, de 7 de novembro de 2011. Dispõe sobre a Política Nacional de Segurança e Saúde no Trabalho - PNSST. [Internet]. Diário Oficial da União 2011 [acessado 2019 jun 25]; 8 nov. Disponível em: http://www.planalto.gov. br/ccivil_03/_ato2011-2014/2011/decreto/ d7602.htm

16. Brasil. Ministério da Saúde. Portaria no 1.823, de 23 de agosto de 2012. Institui a Política Nacional de Saúde do Trabalhador e da Trabalhadora [Internet]. Diário Oficial da União 2012 [acessado 2019 jun 25]; 24 ago. Disponível em: http://bvsms.saude.gov.br/bvs/ saudelegis/gm/2012/prt1823_23_08_2012. html 
17. Governo do Estado de Mato Grosso. Coordenadoria de Saúde do Trabalhador. O que é Cerest. [Internet]. [acessado $2020 \mathrm{fev} 14$ ]. Disponível em: http://www.saude.mt.gov.br/ cosat/pagina/251/o-que-e-cerest

18. Secretaria de Saúde do estado do Paraná. O que são CEREST's. [Internet]. [acessado 2020 fev 14]. Disponível em: http://www. saude.pr.gov.br/modules/conteudo/conteudo.php?\%20 conteudo=331

19. Brasil. Ministério do Trabalho. Portaria $n^{\circ}$ 3.214 , de 8 de junho de 1978. Aprova as Normas Regulamentadoras - NR - do Capítulo V, Título II, da Consolidação das Leis do Trabalho, relativas à Segurança e Medicina do Trabalho. [Internet]. Diário Oficial da União 1978 [acessado 2020 fev 14]; 6 jul. Disponível em: http://www.normaslegais.com.br/legislacao/trabalhista/portariamte3214.htm

20. Alvares JK, Pinheiro TMM, Santos AF, Oliveira GL. Avaliação da completitude das notificações compulsórias relacionadas ao trabalho registradas por município polo industrial no Brasil, 2007 - 2011. Rev Bras Epidemiol 2015; 18(1):123-36.

21. Fundacentro. Brasil registra 17 mil mortes e 4 milhões de acidentes de trabalho. [Internet]. 2019 [acessado 2020 fev 14]. Disponível em: http://www.fundacentro.gov.br/noticias/ detalhe-da-noticia/2019/4/acoes-regressivasgestao-de-riscos-e-impacto-dos-acidentesde-trabalho-foram-temas-de-debate

22. Leite PC, Silva A, Merighi MAB. A mulher trabalhadora de enfermagem e os distúrbios osteomusculares relacionados ao trabalho. Rev Esc Enferm USP 2007; 41(2):287-91.

23. Brasil. Ministério da Economia. Instituto Nacional do Seguro Nacional. Comunicação de Acidente de Trabalho - CAT. [Internet]. 2018 [acessado 2020 fev 14]. Disponível em: https://www.inss.gov.br/servicos-do-inss/ comunicacao-de-acidente-de-trabalho-cat/

24. Pontes HMM. Pesquisa revela retrato inédito do mercado de trabalho do interior do país [Internet]. 2019 [acessado $2020 \mathrm{fev}$ 14]. Disponível em: https://agenciadenoticias.ibge. gov.br/agencia-noticias/2012-agencia-de-noticias/noticias/25066-pesquisa-revela-retrato-inedito-do-mercado-de-trabalho-do-interior-do-pais

25. Brasil. Ministério da Saúde. Portaria no 104, de 25 de janeiro de 2011. Define as terminologias adotadas em legislação nacional, conforme o disposto no Regulamento Sanitário Internacional 2005 (RSI 2005), a relação de doenças, agravos e eventos em saúde pública de notificação compulsória em todo o território nacional e estabelece fluxo, critérios, responsabilidades e atribuições aos profissionais e serviços de saúde. [Internet]. Diário Oficial da União 2011 [acessado 2020 fev 14]; 26 jan. Disponível em: http:// bvsms.saude.gov.br/bvs/saudelegis/gm/2011/ prt0104_25_01_2011.html

26. Brasil. Ministério da Saúde. Portaria no 204, de 17 de fevereiro de 2016. Define a Lista Nacional de Notificação Compulsória de doenças, agravos e eventos de saúde pública nos serviços de saúde públicos e privados em todo o território nacional, nos termos do anexo, e dá outras providências. [Internet]. Diário Oficial da União 2016 [acessado 2019 jun 25]; 18 fev. Disponível em: http:// bvsms.saude.gov.br/bvs/saudelegis/gm/2016/ prt0204_17_02_2016.html

27. Medina FS, Maia MZB. A subnotificação de LER/DORT sob a ótica de profissionais de saúde de Palmas, Tocantins. Rev Bras Saúde Ocupacional [Internet] 2016 [acessado 2019 jun 25]; 41(0). Disponível em: http://www. scielo. 
28. Ministério da Saúde. Política Nacional de Segu- 30. Dias EC, Silva TL, Chiavegatto CV, Reis JC, rança e Saúde do Trabalhador. [Internet]. 2004 Campos AS. Desenvolvimento de ações de [acessado 2020 fev 14]. Disponível em: http:// saúde do trabalhador no SUS: a estratégia bvsms.saude.gov.br/bvs/publicacoes/politica_ nacional_seguranca_saude.pdf

29. Cavalcante CAA, Santos RS, Cavalcante EFO, Martins RL, Silveira EA, Silva ET. Perfil dos agravos relacionados ao trabalho notificados no Rio Grande do Norte, 2007 a 2009. Epidemiol Serv Saúde 2014; 23(4):741-52.

30. Dias EC, Silva TL, Chiavegatto CV, Reis JC, Campos AS. Desenvolvimento de ações de saúde do trabalhador no SUS: a estratégia da Rede Nacional de Atenção Integral à Saúde do Trabalhador (RENAST) [Internet]. Editora Fiocruz; 2011 [acessado $2020 \mathrm{fev}$ 14]. Disponível em: http://bases.bireme.br/cgi-bin/ wxislind.exe/iah/online/?IsisScript=iah/iah. xis\&src $=$ google \&base $=$ LILACS\&lang $=$ p\&nextAction $=\operatorname{lnk} \& \operatorname{exprSearch}=638196 \&$ indexSearch $=$ IDbr/scielo.php?script $=$ sci_arttext\&pid=S0303-76572016000100206\&lng=pt\&tl$\mathrm{ng}=\mathrm{pt}$

28. Ministério da Saúde. Política Nacional de Segurança e Saúde do Trabalhador. [Internet]. 2004 [acessado 2020 fev 14]. Disponível em: http://bvsms.saude.gov.br/bvs/publicacoes/ politica_nacional_seguranca_saude.pdf

29. Cavalcante CAA, Santos RS, Cavalcante EFO, Martins RL, Silveira EA, Silva ET. Perfil dos agravos relacionados ao trabalho notificados da Rede Nacional de Atenção Integral à Saúde do Trabalhador (RENAST) [Internet]. Editora Fiocruz; 2011 [acessado 2020 fev 14]. Disponível em: http://bases.bireme. br/cgi-bin/wxislind.exe/iah/online/?IsisScript $=$ iah $/$ iah. $x i s \& s r c=$ google $\&$ base $=$ LI LACS\&lang $=$ p\&nextAction $=\operatorname{lnk} \& \operatorname{expr}-$ Search=638196\&indexSearch=ID

Recebido: 05/10/2019 Aprovado: 07/05/2020 no Rio Grande do Norte, 2007 a 2009. Epidemiol Serv Saúde 2014; 23(4):741-52. 